\title{
RELAÇÃO ENTRE FORÇA MUSCULAR RESPIRATÓRIA E DE PREENSÃO PALMAR EMIDOSOS INSTITUCIONALIZADOS E DA COMUNIDADE
}

\section{Relationship between respiratory muscle strength and grip strength in institutionalized and community-dwelling older adults}

\author{
Liliane de Faria Marcon ${ }^{a}\left(\mathbb{D}\right.$, Ruth Caldeira de Melo ${ }^{\mathrm{a}}$, Francisco Luciano Pontes Júniora ${ }^{\mathrm{C}}$
}

OBJETIVO: Avaliar a relação entre força muscular respiratória e força de preensão palmar em idosos institucionalizados e da comunidade. METODOLOGIA: Caracteriza-se por um estudo transversal com 64 voluntários, sendo 33 institucionalizados e 31 da comunidade. Avaliou-se a pressão inspiratória máxima, pressão expiratória máxima, pico de fluxo expiratório, força de preensão palmar, dados antropométricos e nível de atividade física. Os dados foram submetidos a uma análise descritiva, teste de correlação de Pearson e análise de regressão linear. RESULTADOS: No grupo de institucionalizados, não houve correlação entre as variáveis respiratórias e as de força de preensão palmar, porém o preditor respiratório mais fortemente associado à força de preensão palmar foi a pressão expiratória máxima ( $p=0,04)$. No grupo da comunidade, verificou-se correlação entre pressão inspiratória máxima e força de preensão palmar $(r=0,54)$, pressão expiratória máxima e força de preensão palmar $(r=0,62)$, pico de fluxo expiratório e força de preensão palmar $(r=0,64)$, e o preditor respiratório mais fortemente associado à força de preensão palmar foi pico de fluxo expiratório e pressão expiratória máxima $(p<0,05)$. Na análise conjunta dos grupos, verificou-se associação entre pressão inspiratória máxima e força de preensão palmar $(r=0,40)$, pressão expiratória máxima e força de preensão palmar $(r=0,57)$, pico de fluxo expiratório e força de preensão palmar ( $r=0,57)$, e o preditor respiratório mais fortemente associado à força de preensão palmar foi pico de fluxo expiratório e pressão expiratória máxima $(p<0,05)$. CONCLUSÕES: Em idosos da comunidade, o pico de fluxo expiratório e a pressão expiratória máxima parecem ser bons preditores da força de preensão palmar, enquanto, em institucionalizados, essa relação parece não se manter possivelmente por conta de maior acentuação de perda da função respiratória.

PALAVRAS-CHAVE: músculos respiratórios; força muscular; idoso.

OBJECTIVE: To evaluate the relationship between respiratory muscle strength and grip strength in institutionalized and communitydwelling older adults. METHODS: This convenience sampled cross-sectional study had 64 voluntary participants with a good general health status and without limiting chronic diseases: 33 were institutionalized and 31 lived in the community. Maximal inspiratory pressure, maximal expiratory pressure, peak expiratory flow, grip strength, anthropometric data, and physical activity level were assessed. The data were subjected to descriptive analysis, Pearson's correlation test, and linear regression analysis. RESULTS: In the institutionalized group, there was no between respiratory variables and grip strength, but maximal expiratory pressure was the respiratory predictor most strongly associated with grip strength $(p=0.04)$. In the community-dwelling group, there was a correlation between maximal inspiratory pressure and grip strength $(r=0.54)$, maximal expiratory pressure and grip strength $(r=0.62)$, and peak expiratory flow and grip strength $(r=0.64)$; peak expiratory flow and maximal expiratory pressure were the respiratory predictors most strongly associated with grip strength $(p<0.05)$. In a joint group analysis, there was an association between maximal inspiratory pressure and grip strength $(r=0.40)$, maximal expiratory pressure and grip strength $(r=0.57)$, and peak expiratory flow and grip strength $(r=0.57)$; peak expiratory flow and maximal expiratory pressure were the respiratory predictors most strongly associated with grip strength $(p<0.05)$. CONCLUSIONS: Peak expiratory flow and maximal expiratory pressure seem to be good predictors of grip strength in community-dwelling older adults, but this relationship does not seem to be maintained in institutionalized patients, possibly because of a greater loss of respiratory function.

KEYWORDS: respiratory muscles; muscle strength; aged.

aPrograma de Pós-Graduação em Gerontologia, Laboratório de Fisiologia do Exercício e Envelhecimento, Escola de Artes, Ciências e Humanidades, Universidade de São Paulo - São Paulo (SP), Brasil.

Dados para correspondência

Liliane de Faria Marcon - Avenida Voluntário Benedito Sergio, 1.085 - Estiva - CEP: 12050-470 - Taubaté (SP), Brasil. E-mail: liliane.marcon@alumni. usp.br @MarconLiliane

Recebido em: 30/11/2020. Aceito em: 09/03/2021

Como citar este artigo: Marcon LF, Melo RC, Pontes Júnior FL. Relação entre força muscular respiratória e de preensão palmar em idosos institucionalizados e da comunidade. Geriatr Gerontol Aging. 2021;15:e0210012. https://doi.org/10.5327/Z2447-212320212000148

https://doi.org/10.5327/22447-212320212000148

Este artigo é publicado em Acesso Aberto sob a licença Creative Commons Attribution, que permite o uso, distribuição e reprodução em qualquer meio, sem restrições, desde que o trabalho original seja corretamente citado. 


\section{INTRODUÇÃO}

A estrutura e a função do sistema pulmonar mudam significativamente entre a fase adulta e o envelhecimento. Entre as diversas alterações que ocorrem neste sistema, as diminuições da complacência torácica, do recuo elástico pulmonar e da força dos músculos respiratórios estão associadas com prejuízos funcionais dos idosos, pela modificação na concentração de colágeno, elastina e cálcio, além das modificações estruturais da coluna vertebral. ${ }^{1-3}$

Adicionalmente, a inervação simpática diminuída das vias aéreas pode aumentar o estado de constrição, contribuindo para a diminuição do fluxo aéreo, parâmetro este que pode ser medido pelo pico de fluxo expiratório (PFE) por ser uma medida simples e de baixo custo na indisponibilidade de espirômetro. ${ }^{2,4}$

As pressões inspiratórias e expiratórias máximas (PImax e PEmax), que refletem a força muscular respiratória, diminuem com o envelhecimento, porém parece não haver alteração na espessura do diafragma com a idade e sim uma desvantagem mecânica advinda da retificação da sua cúpula e da redução da condução nervosa do nervo frênico. 3,5

Em contrapartida, o processo de envelhecimento também é acompanhado por alterações no sistema musculoesquelético que resultam em redução da força e da massa muscular, sendo a força muscular o critério de maior impacto e que também pode ser fortemente influenciado pelo nível de atividade física. ${ }^{6}$

Se, por um lado, a sarcopenia periférica predispõe os idosos a eventos negativos, tais como fragilidade, institucionalização e morte, ${ }^{6-8}$ por outro, a fraqueza muscular respiratória predispõe a complicações respiratórias que indiretamente podem potencializar os riscos desses desfechos. ${ }^{9}$

Poucos estudos anteriores demonstram que a perda de força periférica e da musculatura respiratória pode ter uma mesma origem, comprometendo a funcionalidade tanto pela disfunção respiratória como pela incapacidade motora, ainda mais em idosos institucionalizados que já têm maior perfil de fragilidade e maiores índices de sarcopenia. ${ }^{1,10-13}$

Nossa hipótese é que existe uma relação entre a força muscular respiratória e a força periférica em idosos e, visto que o método de avaliação de força periférica é simples e tem maior disponibilidade no sistema de saúde do que o recurso de avaliação respiratória, a detecção de fraqueza periférica poderia direcionar os casos que precisam de avaliação da função respiratória. Com base nesse delineamento, seria possível a implementação de estratégias preventivas e de tratamento, como a fisioterapia respiratória.

Dessa forma, foi objetivo deste estudo investigar a existência de relação entre força muscular respiratória e força de preensão palmar (FPP) em idosos institucionalizados e da comunidade, já que não foram encontrados estudos comparativos entre esses dois públicos.

\section{METODOLOGIA}

Este trabalho se trata de estudo quantitativo com delineamento transversal. A amostra de conveniência foi composta de 64 voluntários, de ambos os sexos, com idade igual ou superior a 60 anos, sendo 33 residentes em instituições de longa permanência para idosos (ILPIs) filantrópicas do Vale do Paraíba (SP) (GI) e 31 residentes na comunidade (GC). Todos os voluntários que aceitaram participar da pesquisa assinaram o termo de consentimento informado livre e esclarecido de acordo com a Resolução CNS no 466/2012, após ele ter sido aprovado pelo Comitê de Ética em Pesquisa em Seres Humanos da Escola de Artes, Ciências e Humanidades da Universidade de São Paulo (no 1.890.933).

Os critérios de inclusão para os grupos estudados foram: apresentar bom estado geral de saúde e não apresentar doenças crônicas limitantes. Essas condições foram avaliadas por meio dos prontuários dos idosos institucionalizados e autorrelatadas nos idosos da comunidade. Excluíram-se os idosos com distúrbios psiquiátricos, mentais e neurológicos, doença pulmonar, neuropatia de aprisionamento médio, tabagista ativo, cegueira, surdez, hospitalização nos últimos 6 meses e com dificuldade para compreender e realizar corretamente os procedimentos.

\section{Avaliação antropométrica e do nível de atividade física}

Além do registro da idade e do sexo, a caracterização antropométrica da amostra foi realizada com base na medida do peso e a estatura por meio de uma balança mecânica Welmy (110CH, Brasil) e um estadiômetro da marca Sanny (ES2030, Brasil). O nível de atividade física foi avaliado pelo Questionário Internacional de Atividade Física (IPAQ). Esse é composto de cinco domínios e 27 questões e classifica o perfil de atividade física em: muito ativo, ativo, irregularmente ativo e sedentário. ${ }^{14}$

\section{Avaliação da força muscular respiratória}

A força muscular respiratória foi avaliada por meio das PImax e PEmax, de acordo com a American Thoracic Society/ European Respiratory Society (ATS/ERS). ${ }^{15}$ Para tanto, utilizou-se um manovacuômetro analógico Globalmed (M120, Brasil) graduado de 0 até $120 \mathrm{cmH}_{2} \mathrm{O}$. O voluntário foi posicionado sentado com o tronco ereto e orientado a realizar duas manobras para aprendizado. Para medida de PImax, utilizou-se como ponto inicial de coleta a expiração máxima (volume de reserva expiratório - VRE) seguida de uma inspiração 
máxima.Já para a medida de PEmax, utilizou-se como ponto inicial de coleta a capacidade pulmonar total (CPT) e, depois, solicitou-se uma expiração máxima. A posição alcançada ao fim dos esforços máximos deveria ser mantida por, pelo menos, um segundo para caracterização da pressão de platô. Realizaram-se cinco manobras, sendo três aceitáveis, com, pelo menos, duas manobras reprodutíveis. Entre cada manobra, houve um intervalo de repouso de um minuto.

\section{Avaliação do pico de fluxo expiratório}

A mensuração do PFE foi feita por meio do aparelho Peak Flow Meter (Clement Clarke, AirZone, Inglaterra). Solicitou-se ao idoso que mantivesse a posição ortostática com uso do clipe nasal e, após uma inspiração máxima, realizasse uma expiração rápida e forçada no bucal, conforme determinação da ATS/ETS. ${ }^{15}$ Foram feitas três aferições, com intervalos de 30 segundos, sendo anotado o maior valor encontrado desde que as mesmas medidas não variassem em mais de 40 litros entre elas.

\section{Avaliação da força de preensão palmar}

Utilizou-se um dinamômetro de preensão palmar (SAEHAN hidráulico, 5001, Coreia do Sul), ${ }^{16}$ o qual possui um sistema hidráulico de duas alças paralelas. O dinamômetro mede a força de uma contração máxima, sendo registrada em quilogramas. O teste foi aplicado no membro dominante, estando o voluntário sentado com seu cotovelo flexionado em $90^{\circ}$ e o antebraço e o punho mantidos em posição neutra. Realizaram-se cinco medidas, sendo feita a média dos valores.

A análise estatística utilizou o software SPSS 23. Inicialmente, realizou-se a análise descritiva dos dados, incluindo medidas de tendência central, dispersão e análise da distribuição dos dados, teste de Levene e teste de Shapiro-Wilk.

Para as variáveis referentes às características dos sujeitos, os grupos foram comparados entre si pelo teste $t$ de Student para amostras independentes. Já para as variáveis respiratórias e para a FPP, os grupos foram comparados pela análise de covariância (ANCOVA) controlada pela covariável idade, uma vez que os grupos eram diferentes entre si. $\mathrm{O}$ tamanho de efeito entre os grupos foi analisado por meio do teste $\mathrm{d}$ de Cohen. Foi estabelecido IC95\% e nível de significância de $\mathrm{p}<0,05$.

A verificação da correlação utilizou o teste de correlação de Pearson em que 0,70 a 0,90 indica uma correlação forte, de 0,50 a 0,70 indica uma correlação moderada e de 0,30 a 0,50 indica uma correlação fraca. ${ }^{17}$ Além disso, estabeleceu-se o tamanho de efeito para avaliar a existência de diferenças significativas entre as correlações entre os grupos. Para retirar a probabilidade de erro do tipo I, utilizou-se a correção de Bonferroni, sendo estabelecido o nível de significância de $\mathrm{p}<0,005$. Adicionalmente, uma análise de regressão linear pelo método Enter foi empregada para a verificação de influência das variáveis respiratórias sobre a FPP.

\section{RESULTADOS}

\section{Caracterização da amostra}

O GI apresentou maior idade, com diferenças significativas $(76,73 \pm 7,96)$ em comparação ao $\operatorname{GC}(69,27 \pm 6,83)$. Em relação ao peso, o grupo GI apresentou menor peso corporal, com significativa diferença $(62,40 \pm 14,98)$ em comparação ao GC $(72,06 \pm 14,03)$. Para as variáveis estatura e índice de massa corporal (IMC), não foram encontradas diferenças estatisticamente significantes com d de Cohen de $-0,48$ e probabilidade de $63,30 \%$ das mesmas médias serem menores no GI (Tabela 1). A proporção de idosos do sexo feminino foi de $60,60 \%$ para o GI e de $74,20 \%$ para o GC.

O teste da ANCOVA descartou o efeito da idade sobre as variáveis respiratórias e força de preensão manual. Em contrapartida, ele demonstrou efeito de grupo para elas, em que

Tabela 1. Características de idosos institucionalizados $(n=33)$ e residentes na comunidade $(n=31)$, Vale do Paraíba, São Paulo, Brasil, 2018.

\begin{tabular}{l|c|c|c|c|c|c}
\multirow{2}{*}{ Idade } & \multicolumn{2}{|c|}{ GI } & \multicolumn{2}{c}{ GC } & \multirow{2}{*}{ Valor $p$} & \multirow{2}{*}{ Tamanho de efeito } \\
\cline { 2 - 6 } & Média & Desvio padrão & Média & Desvio padrão & \multicolumn{2}{c}{1,00} \\
\hline Peso & $76,73^{*}$ & 7,96 & 69,27 & 6,83 & $<0,001$ & $-0,66$ \\
\hline Altura & 62,40 & 14,98 & 72,06 & 14,03 & 0,09 & $-0,48$ \\
\hline IMC & 156,66 & 9,21 & 161,67 & 11,65 & 0,06 & $-0,48$ \\
\hline PFE & 25,31 & 5,19 & 27,73 & 4,86 & 0,05 & $-1,21$ \\
\hline PImax & $193,30^{*}$ & 76,14 & 328,40 & 139,76 & $<0,001$ & $-0,84$ \\
\hline PEmax & $-38,52^{*}$ & 28,09 & $-63,11$ & 30,28 & 0,001 & 21,11 \\
\hline FPP & $52,17^{*}$ & 20,42 & 75,61 & 21,76 & $<0,001$ & $-0,72$ \\
\hline
\end{tabular}

${ }^{*} \mathrm{p}<$ 0,05; IMC: índice de massa corporal; PFE: pico de fluxo expiratório; PImax: pressão inspiratória máxima; PEmax: pressão expiratória máxima; FPP: força de preensão palmar; Gl: idosos institucionalizados; GC: idosos da comunidade. 
Tabela 2. Distribuição de idosos institucionalizados ( $\mathrm{n}=$ $33)$ e residentes na comunidade $(n=31)$ segundo nível de atividade física do IPAQ, Vale do Paraíba, São Paulo, Brasil, 2018.

\begin{tabular}{l|c|c} 
Muito ativo & GI $(\%)$ & GC $(\%)$ \\
\hline Ativo & & $1(3,20)$ \\
\hline Insuficientemente ativo & $5(17,60)$ & $16(51,60)$ \\
\hline Sedentário & $15(44,10)$ & $2(6,50)$ \\
\hline
\end{tabular}

IPAQ: Questionário Internacional de Atividade Física; GI: idosos institucionalizados; GC: idosos da comunidade.

Tabela 3. Coeficiente de correlação de Pearson entre as variáveis respiratórias e de força de preensão em idosos institucionalizados ( $n=33$ ), residentes na comunidade ( $n$ = 31) e amostra total ( $n=64)$, Vale do Paraíba, São Paulo, Brasil, 2018.

\begin{tabular}{l|c|c|c|c} 
GI $(\mathbf{n}=33)$ & PFE & PImax & PEmax & FPP \\
\hline PFE & 1,00 & 0,19 & 0,23 & 0,39 \\
\hline PImax & & 1,00 & $0,59^{*}$ & 0,13 \\
\hline PEmax & & & 1,00 & 0,40 \\
\hline FPP & & & & 1,00 \\
\hline GC $(n=31)$ & PFE & PImax & PEmax & FPP \\
\hline PFE & 1,00 & $0,56^{*}$ & 0,46 & $0,64^{*}$ \\
\hline PImax & & 1,00 & $0,67^{*}$ & $0,53^{*}$ \\
\hline PEmax & & & 1,00 & $0,62^{*}$ \\
\hline FPP & & & & 1,00 \\
\hline GI + GC $(n=$ & PFE & PImax & PEmax & FPP \\
\hline 64) & 1,00 & $0,53^{*}$ & $0,53^{*}$ & $0,57^{*}$ \\
\hline PFE & & 1,00 & $0,70^{*}$ & $0,40^{*}$ \\
\hline PImax & & & 1,00 & $0,57^{*}$ \\
\hline PEmax & & & & 1,00 \\
\hline FPP & & & & \\
\hline
\end{tabular}

*Nível de significativa no nível de $p<0,005$ após correção de Bonferroni; PFE: pico de fluxo expiratório; PImax: pressão inspiratória máxima; PEmax: pressão expiratória máxima; FPP: força de preensão palmar; Gl: idosos institucionalizados; GC: idosos da comunidade. todas as variáveis respiratórias (PImax, PEmax e PFE) e a FPP encontraram-se menores no GI.

Verificou-se ainda maior nível de atividade física no GC, com 54,80\% dos voluntários classificados por meio do IPAQ como ativos ou muito ativos, enquanto, no GI, apenas $17,60 \%$ eram ativos. Para ambos os grupos, o percentual de participantes classificados como sedentários foi de aproximadamente $38 \%$ (Tabela 2).

Quando se analisaram as relações entre as variáveis para o GI (Tabela 3), verificou-se que existe correlação moderada positiva e significativa entre as pressões máximas respiratórias PImax vs. PEmax (0,59), porém não foi encontrada correlação entre variáveis respiratórias e de preensão. No GC, observou-se correlação moderada positiva e significativa entre as variáveis PFE vs. PImax (0,64) e PFE vs. FPP (0,64), PImax vs. PEmax $(0,67)$ e PEmax vs. FPP $(0,62)$. Na análise sem discriminação de grupos $(\mathrm{GI}+\mathrm{GC}=\mathrm{GT})$, houve correlações moderadas, positivas e significativas entre PImax vs. PFE (0,53), PImax vs. PEmax (0,70), PEmax vs. FPP $(0,57)$.

Da análise de regressão linear simples para o GI (Tabela 4), há significância estatística do indicador PEmax para a FPP $\left(\mathrm{R}^{2}=0,27, \mathrm{~F}_{(3,29)}=3,69 ; \mathrm{p}=0,023 ; \mathrm{DW}=1,88\right)$. Em relação ao $\mathrm{GC}$, os seguintes indicadores em ordem de importância da estatística F foram PFE e PEmax para FPP $\left(\mathrm{R}^{2}=0,74\right.$; $\left.\mathrm{F}_{(3,27)}=10,96 ; \mathrm{p}<0,001 ; \mathrm{DW}=1,86\right)$. Quando analisada a junção da amostra, verificou-se significância estatística dos indicadores PFE e PEmax para a FPP $\left(\mathrm{R}^{2}=0,44, \mathrm{~F}_{(3,60)}=\right.$ $15,76, \mathrm{p}<0,05 ; \mathrm{DW}=1,97)$.

\section{DISCUSSÃO}

$\mathrm{O}$ perfil dos idosos do GI diferiu do GC em relação à força muscular periférica e à força da musculatura respiratória. Esses fatores podem ter sido influenciados por diferentes aspectos, como o perfil de realização de atividade física e uma característica mais fragilizada e com possibilidade de sarcopenia, visto que, no GI, encontramos valores de FPP inferiores ao critério descrito pelo European Working Group on

Tabela 4. Análise de regressão linear para variáveis respiratórias que predizem a força de preensão em idosos institucionalizados ( $n=33)$, residentes na comunidade $(n=31)$ e amostra total $(n=64)$, Vale do Paraíba, São Paulo, Brasil, 2018.

\begin{tabular}{|c|c|c|c|c|c|c|}
\hline \multicolumn{7}{|c|}{ FPP } \\
\hline & \multicolumn{2}{|c|}{ GI } & \multicolumn{2}{|c|}{ GC } & \multicolumn{2}{|c|}{$\mathbf{G I}+\mathbf{G C}$} \\
\hline & $\beta$ & Valor $p$ & $\beta$ & Valor $p$ & $\beta$ & Valor $p$ \\
\hline PFE & 0,042 & 0,05 & 0,025 & $0,009 *$ & 0,409 & $0,01^{*}$ \\
\hline PImax & $-0,061$ & 0,37 & 0,002 & 0,96 & $-0,120$ & 0,39 \\
\hline PEmax & 0,203 & $0,04^{*}$ & 0,149 & $0,02^{*}$ & 0,440 & $0,03^{*}$ \\
\hline
\end{tabular}

* $\mathrm{p}$ < 0,05; $\beta$ : beta; PFE: pico de fluxo expiratório; PImax: pressão inspiratória máxima; PEmax: pressão expiratória máxima; FPP: força de preensão palmar; Gl: idosos institucionalizados; GC: idosos da comunidade. 
Sarcopenia in Older People 2 (EWGSOP2), ${ }^{9}$ principalmente na população masculina $(\mathrm{FPP}=25 \mathrm{~kg})$, porém não foram avaliadas a massa muscular e o desempenho físico para que esse determinante pudesse ser confirmado. Em um estudo conduzido por Vidal et al., ${ }^{18}$ eles sugerem um ponto de corte como marcador de fragilidade por meio da PImax $(>-50 \mathrm{~cm}$ $\left.\mathrm{H}_{2} \mathrm{O}\right)$ e $\mathrm{PEmax}\left(<60 \mathrm{~cm} \mathrm{H} \mathrm{H}_{2} \mathrm{O}\right)$ e de pré-fragilidade para a PEmax $\left(<65 \mathrm{~cm} \mathrm{H}_{2} \mathrm{O}\right)$. Embora a fragilidade não tenha sido foco do nosso estudo, se considerarmos esses critérios respiratórios, verificamos que o GI apresenta um perfil com capacidade funcional mais reduzida do que o GC e pode ser foco de futuros estudos clínicos.

Essas características vêm ao encontro do descrito por Fragoso et al., ${ }^{19}$ que relatam que a fraqueza muscular respiratória é prevalente em idosos sedentários e está associada à inatividade física, além de associar-se à maior probabilidade de hospitalização por causas respiratórias. A presença de idosos mais frágeis no GI também precisa ser considerada, já que o menor peso corporal associado a baixos índices de FPP e baixo nível de atividade física são marcadores importantes de fragilidade. Para Vaz Fragoso et al., ${ }^{20}$ os idosos que apresentam fraqueza da musculatura respiratória aumentam em até quatro vezes a incidência de mortalidade, e a relação entre declínio respiratório e fragilidade é bidirecional, podendo um conduzir ao outro. Ao detectar a fraqueza muscular respiratória, o encaminhamento para programas de fisioterapia respiratória e atividade física poderá auxiliar na manutenção e no ganho dessa força, como demonstrado por Souza et al., ${ }^{21}$ que, no acompanhamento por oito semanas de treinamento respiratório de intensidade moderada, encontraram aumento de força, espessura e mobilidade do diafragma.

Partindo do pressuposto que o GI apresenta características de fragilidade e sarcopenia, a inexistência da correlação entre as variáveis de força respiratória e periférica nesse grupo, apesar da relação existir em idosos da comunidade e na amostra geral, pode ser explicada pela perda mais acentuada da força muscular respiratória do que da força periférica, evoluindo de maneira diferente com relação aos seguimentos estudados, já que o GI tem maior perda de força em relação ao valor predito (51\% da PImax e 36\% da PEmax) do que a força periférica ( $24 \%$ da FPP).

Vale ressaltar que os homens do GI apresentaram perda de força periférica e respiratória mais evidente do que as mulheres, sendo esses dados também sustentados por Ritti-Dias et al. ${ }^{22} \mathrm{em}$ um estudo com 288 idosos. Por sua vez, Bahat et al. ${ }^{23}$ estudaram um grupo de 104 homens idosos residentes de ILPI e argumentaram que a sarcopenia pode afetar a força muscular inspiratória mais precocemente ou em maior intensidade do que a força muscular expiratória. Provavelmente o idoso com maior perfil de fragilidade preserva as fibras oxidativas do diafragma a fim de manter a função ventilatória essencial, mas não as fibras de contração rápida (IIb e IIx) que são recrutadas ante atividades não ventilatórias, como a tosse. Isso foi amparado por Elliott et al..$^{24} \mathrm{em}$ um estudo conduzido em modelo animal, em que se identificou redução de aproximadamente $20 \%$ desses tipos de fibras e $24 \%$ da força máxima.

Quando avaliados os dados de idosos da comunidade (GC), houve correlação positiva e moderada entre força muscular respiratória e FPP, provavelmente atribuída à maior preservação de ambas as funções do que aqueles observados no GI. Esses dados foram suportados por Enright et al., ${ }^{25}$ que também verificaram correlação de PImax e PEmax com FPP em uma coorte de idosos não institucionalizados e declararam que os efeitos do envelhecimento ocorrem de maneira similar em ambos os grupos musculares, e por Shin et al., ${ }^{26}$ que afirmam existir uma correlação ainda mais forte com a PImax. Porém, Costes et al., ${ }^{27}$ apesar de encontrarem correlação fraca entre PImax e FPP, afirmam que os determinantes da redução da força inspiratória máxima diferiram dos determinantes da FPP, sendo esta relacionada à força do tronco em homens, aspecto não estudado por nós, porém sabidamente podendo sofrer influência das alterações mecânicas do tórax ocasionadas pelo envelhecimento.

Em um estudo conduzido por Pegorari et al. ${ }^{28}$, eles encontraram uma correlação positiva forte entre PImax e PEmax com a FPP em idosos pré-frágeis da comunidade. Em outro estudo, com o objetivo de avaliar os índices sarcopênicos em relação à força muscular respiratória de idosos da comunidade, os autores encontraram correlação positiva entre PImax e PEmax com FPP, sugerindo que os músculos respiratórios, especialmente os músculos inspiratórios, estão significativamente relacionados à força muscular dos membros $\mathrm{e}$ à massa muscular esquelética, assim como os nossos dados demonstram..$^{20}$ Logo, a inclusão de programas preventivos de manutenção da capacidade física e funcional podem ser bons aliados para a manutenção da força periférica e respiratória nos idosos da comunidade.

Outro dado analisado foi o PFE, que é utilizado como parâmetro para avaliar a permeabilidade das vias aéreas bem como para avaliação indireta da força muscular expiratória forçada, em que há ativação dos músculos abdominais e intercostais internos. Para nós, esse marcador foi um importante achado, já que poucos estudos utilizaram o $\mathrm{PFE}$ como instrumento de análise, servindo como um marcador preditivo da FPP no GC. Vale ressaltar que esse grupo apresentou menor redução das forças periféricas e respiratórias bem como maior nível de atividade física, podendo este ter 
contribuído para maior preservação da função muscular, já que, na estratificação pela idade, não tivemos influência desta sobre o nosso resultado.

O PFE no GI teve valores menores do que o predito além de não ter sido verificada correlação entre o PFE e a FPP, sugerindo que os idosos desse grupo apresentam uma perda mais acentuada da musculatura inspiratória do que da expiratória forçada e maior resistência das vias aéreas. Vaz Fragoso et al. ${ }^{20}$ identificaram que os idosos caracterizados como pré-frágeis e frágeis obtiveram maior probabilidade de ter limitação ao fluxo aéreo e de ter um padrão restritivo. Para Bahat et al., ${ }^{23}$ o PFE em homens institucionalizados foi um preditor da FPP, diferentemente dos nossos achados.

A ausência de correlação no GI pode ter ocorrido pela maior porcentagem de idosos sedentários, podendo influenciar a capacidade de geração de força e fluxo expiratório dada a baixa reserva energética e contribuindo para uma pobre oxigenação da musculatura esquelética periférica. Ritti-Dias et al..$^{22}$ estudaram uma população mista, advinda da comunidade e de ILPI, e também encontraram correlação tanto em homens como em mulheres, porém relatam que os mecanismos explicativos ainda são obscuros. Além disso, também avaliaram a mobilidade e identificaram que, em mulheres, o PFE intermedeia a relação entre a FPP e a mobilidade. Sllanpää et al. ${ }^{1}$ sugerem que o declínio na mobilidade é regulado diretamente por meio da diminuição da força e da potência muscular, mas também parcialmente mediado por diminuição da função pulmonar em idosos saudáveis. Kera et al. ${ }^{29}$ definiram o termo sarcopenia respiratória e determinaram um valor de corte para homens $(4,40 \mathrm{~L} / \mathrm{s})$ e mulheres $(3,21 \mathrm{~L} / \mathrm{s})$ idosos. Esses critérios podem contribuir para a utilização desses dados na prática clínica.

A falta de avaliação do grau de obstrução das vias aéreas pela espirometria pode ser um ponto limitante. Para trabalhos posteriores, sugerimos estudos longitudinais que permitam identificar aspectos de causa e efeito dessa relação em idosos institucionalizados e da comunidade. Ainda sugerimos estudos com propostas de intervenções preventivas ou que visem diminuir as complicações advindas desse processo de declínio respiratório até aqui conhecido, já que poucos autores buscaram investigar essa associação nesses grupos específicos.

\section{CONCLUSÃO}

Em conclusão, este estudo demonstra que os idosos institucionalizados apresentam maior fraqueza da musculatura respiratória do que os idosos da comunidade e que as variáveis respiratórias estudadas não se relacionam bem com a FPP no GI, sugerindo que a perda de força respiratória nesta população ocorre mais precocemente do que a FPP. Isso ressalta a importância da inclusão de avaliação respiratória precoce na rotina dos idosos institucionalizados a fim de prevenir complicações respiratórias que possam culminar em internação e óbito, além de implementar programas de atividade física que possam auxiliar na manutenção da força muscular respiratória. Em contrapartida, em idosos da comunidade, essa relação se estabelece e o PFE e a PEmax parecem ser bons preditores para a FPP, fator que contribui para que a avaliação da FPP, que é mais frequentemente empregada na rotina clínica, possa indiretamente inferir a condição respiratória desse idoso.

\section{CONFLITO DE INTERESSES}

Os autores declaram não haver conflito de interesses.

\section{FINANCIAMENTO}

Esta pesquisa não recebeu nenhum financiamento específico de agências de fomento nos setores público, comercial ou sem fins lucrativos.

\section{CONTRIBUIÇÕES DOS AUTORES}

LFM: conceituação, investigação, metodologia, escrita primeira redação. RCM: curadoria de dados, análise formal. FLPJ: Escrita - revisão e edição, supervisão.

\section{REFERÊNCIAS}

1. Sllanpää E, Stenroth L, Bijlma AY, Rantanen T, McPhee JS, MadenWilkinson TM, et al. Association between muscle strength, spirometric pulmonary function and mobility in healthy older adults. Age. 2014;36(4):9667. https://doi.org/10.1007/s1 1357-014-9667-7

2. Brandenberger C, Mühlfeld C. Mechanisms of lung aging. Cell Tissue Res. 2017;367:469-80. https://doi.org/10.1007/s00441-016-2511-x
3. Lalley PM. The aging respiratory system-Pulmonary structure, function and neural control. Respir Physiol Neurobiol. 2013;187(3):199210. https://doi.org/10.1016/j.resp.2013.03.012

4. Fragoso CA, Gill TM. Respiratory Impairment and the Aging Lung. J Gerontol A Biol Sci Med Sci. 2012;67A(3):264-75. https://doi. org/10.1093/gerona/glr198 
5. Neder JA, Andreoni S, Lerario MC, Nery LE. Reference values for lung function tests. II. Maximal respiratory pressures and voluntary ventilation. Braz J Med Biol Res. 1999;32(6):719-27. https://doi. org/10.1590/S0100-879X1999000600007

6. Cruz-Jentoft AJ, Bahat G, Bauer J, Boirie Y, Brurière O, Cederholm $\mathrm{T}$, et al. Sarcopenia: revised European consensus on definition and diagnosis. Age Ageing. 2019;48(1):16-31. https://doi.org/10.1093/ ageing/afy169

7. Mclean RR, Kiel DP. Developing Consensus Criteria for Sarcopenia: An Update. J Bone Miner Res. 2015;30(4):588-92. https://doi. org/10.1002/jbmr.2492

8. Fried LP, Tangen CM, Walston J, Newman AB, Hirsch C, Gottdiener J, et al. Frailty in Older Adults: Evidence for a Phenotype. J Gerontol Med Sci. 2001;56A(3):M146-M156. https://doi.org/10.1093/gerona/56.3.m146

9. Fitting JW. Volitional assessment of respiratory muscle strength. Monaldi Arch Chest Dis. 2012;77(1):19-22. https://doi.org/10.4081/ monaldi.2012.162

10. Landi F, Liperoti R, Fusco D, Mastropaolo S, Quattrociocchi D, Proia A, et al. Sarcopenia and Mortality among Older Nursing Home Residents. JAMDA. 2012;13(2):121-6. https://doi.org/10.1016/j. jamda.2011.07.004

11. Mesquita AF, Silva ECD, Eickemberg M, Roriz AKC, Barreto-Medeiro JM, Ramos LB. Factors associated with sarcopenia in institutionalized elderly. Nutr Hosp. 2017;34(2):345-51. https://doi.org/10.20960/nh.427

12. Mijnarends DM, Koster A, Schols JMGA, Meijers JMM, Halfens RJG, Gudnason V, et al. Physical Activity and Incidence of Sarcopenia: The Population-Based AGES - Reykjavik Study. Age Ageing. 2016;45(5):614-20. https://doi.org/10.1093/ageing/afw090

13. Summerhill EM, Angov N, Garber C, McCool FD. Respiratory muscle strength in the physically active elderly. Lung. 2007;185(6):315-20. https://doi.org/10.1007/s00408-007-9027-9

14. Mazo GP, Benedetti TRB. Adaptação do questionário internacional de atividade física para idosos. Rev Bras Cineantropom Desempenho Hum. 2010;12(6):480-4. https://doi.org/10.1590/S198000372010000600013

15. American Thoracic Society/European Respiratory Society. ATS/ERS Statement on respiratory muscle testing. Am J Respir Crit Care Med. 2002;166(4):518-624. https://doi.org/10.1164/rccm.166.4.518

16. Reis MM, Arantes PMM. Medida da força de preensão manual - validade e confiabilidade do dinamômetro saehan. Fisioter Pesqui. 2011;18(2):176-81. https://doi.org/10.1590/S180929502011000200013

17. Mukaka MM. Statistics Corner: A guide to appropriate use of Correlation coefficient in medical research. Malawi Med J. 2012;24(3):69-71.

18. Vidal MB, Pegorari MS, Santos EC, Matos AP, Pinto ACPN, Ohara DG. Respiratory muscle strength for discriminating frailty in community- dwelling elderly: a cross-sectional study. Arch Gerontol Geriatr 2020;89:104082. https://doi.org/10.1016/j.archger.2020.104082

19. Fragoso CAV, Beavers DP, Anton SD, Liu CK, McDermott MM, Newman $A B$, et al. Effect of Structured Physical Activity on Respiratory Outcomes in Sedentary Elderly Adults with Mobility Limitations. J Am Geriatr Soc. 2016;64(3):501-9. https://doi.org/10.1111/jgs.14013

20. Vaz Fragoso CA, Enright P, McAvay G, Van Ness PH, Gill TM. Frailty and respiratory impairment in older persons. Am J Med. 2012;125(1):7986. https://doi.org/10.1016/j.amjmed.2011.06.024

21. Souza H, Rocha T, Pessoa M, Rattes C, Brandão D, Fregonezi G et al. Effects of inspiratory muscle training in elderly women on respiratory muscle strength, diaphragm thickness and mobility. J Gerontol A Biol Sci Med Sci. 2014;69(12):1545-53. https://doi. org/10.1093/gerona/glu182

22. Ritti-Dias RM, Cucato GG, Franco FGM, Cendoroglo MS, Nasri $\mathrm{F}$, Monteiro-Costa ML, et al. Peak expiratory flow mediates the relationship between handgrip strength and timed up and go performance in elderly women, but not men. Clinics. 2016;71(9):51720. https://doi.org/10.6061/clinics/2016(09)06

23. Bahat G, Tufan A, Ozkaya H, Tufan F, Akpinar TS, Akin S, et al. Relation between hand grip strength, respiratory muscle strength and spirometric measures in male nursing home residentes. Aging Male. 2014;17(3):13640. https://doi.org/10.3109/13685538.2014.936001

24. Elliott JE, Omar TS, Mantilla CA, Sieck GC. Diaphragm muscle sarcopenia in Fischer 344 and Brown Norway rats. Exp Physiol. 2016;101(7):883-94. https://doi.org/10.1113/EP085703

25. Enright PL, Kronmal RA, Manolio TA, Schenker MB, Hyatt RE. Respiratory muscle strength in the elderly: Correlates and reference values. Am J Respir Crit Care Med. 1994;149(2 Pt 1):430-8. https:// doi.org/10.1164/ajrccm.149.2.8306041

26. Shin HI, Kim DK, Seo KM, Kang SH, Lee SY, Son S. Relation Between Respiratory Muscle Strength and Skeletal Muscle Mass and Hand Grip Strength in the Healthy Elderly. Ann Rehabil Med. 2017;41(4):68692. https://doi.org/10.5535/arm.2017.41.4.686

27. Costes F, Celle S, Garet M, Kossovsky M, Roche F, Barthélémy JC. Identification and comparison of the predictors of maximal inspiratory force and handgrip in a healthy elderly population. The proof study. Clin Nutr. 2016;35(4):963-7. https://doi.org/10.1016/j.clnu.2015.07.016

28. Pegorari MS, Ruas G, Patrizzi LJ. Relationship between frailty and respiratory function in the community-dwelling elderly. Braz J Phys Ther. 2013;17(1):9-16. https://doi.org/10.1590/S141335552012005000065

29. Kera T, Kawai H, Hirano H, Kojima M, Watanabe Y, Motokawa K, et al. Definition of Respiratory Sarcopenia With Peak Expiratory Flow Rate. J Am Med Dir Assoc. 2019;20(8):1021-5. https://doi. org/10.1016/j.jamda.2018.12.013 